\section{Hsp90 inhibition disrupts JAK-STAT signaling and leads to reductions in splenomegaly in patients with myeloproliferative neoplasms}

The introduction of JAK inhibitors into clinical practice has improved outcomes for patients with myeloproliferative neoplasms (MPNs). Ruxolitinib, the only approved JAK inhibitor for MPN patients, has demonstrated an ability to decrease splenomegaly and relieve constitutional symptoms. ${ }^{1-3}$ However, the effects of JAK inhibitors on disease biology and disease progression are limited. Further, the majority of patients treated with JAK inhibitors demonstrate stability in mutant allele burden, ${ }^{3}$ demonstrating that JAK inhibitors cannot eradicate or markedly attenuate the MPN clone at clinically utilized doses. Examination of the mechanisms that allow MPN cells to persist despite treatment with JAK inhibitors has revealed that exposure to JAK inhibitors initially results in inhibition of the JAK-STAT pathway. However, resistance can occur leading to re-activation of the JAK-STAT pathway, which is mediated by the ability of JAK2 to heterodimerize and be activated in trans by TYK2 and JAK1. ${ }^{4}$ Thus, the continued presence of mutant JAK2 itself allows for re-activation of the JAK-STAT pathway in the setting of ruxolitinib therapy, a finding that suggests that strategies that directly impact JAK2 protein levels may be therapeutically useful. Heat shock protein 90 (Hsp90) is an ATP-dependent protein chaperone involved in regulating the stability of a broad range of proteins, many of which are important in oncogenesis, including JAK2. Previous work from our group and others has demonstrated that JAK2 associates with Hsp90, and that inhibition of Hsp90 leads to degradation of JAK2 and abrogation of JAK-STAT signaling in vitro and in vivo. ${ }^{6,7}$ Further, in vivo treatment of a murine model of myelofibrosis (MF) with an Hsp90 inhibitor resulted in significant reductions in organomegaly, blood count normalization, and a significant survival benefit, when compared to mice treated with placebo. $^{6}$

Based on these pre-clinical observations, we undertook a phase II study of the Hsp90 inhibitor AUY922 (Novartis, transferred to Vernalis), in patients with MPNs, in order to assess the safety, efficacy, and pharmacodynamics of treatment. We conducted an open-label, investigator-initiated single-center phase II trial of AUY922 in patients with: International Prognostic Scoring System (IPSS) ${ }^{8}$ score of 2 or higher primary myelofibrosis (PMF), post-polycythemia vera (PPV-MF) and post-essential thrombocythemia (PET$\mathrm{MF}$ ) myelofibrosis, polycythemia vera (PV), and essential thrombocythemia (ET). Patients who were refractory to, intolerant of, or ineligible for conventional therapy (including ruxolitinib) were eligible for this study (registered at clinicaltrials.gov identifier: 01668173). From 2012 to 2014,

Table 1. Patients' characteristics and outcomes.

\begin{tabular}{|c|c|c|c|c|c|c|c|c|c|}
\hline Subject & Age & Diagnosis & $\begin{array}{c}\text { Prior } \\
\text { therapies }\end{array}$ & $\begin{array}{l}\text { Baseline } \\
\text { mutations }\end{array}$ & $\begin{array}{l}\text { Duration } \\
\text { of therapy } \\
\text { (months) }\end{array}$ & $\begin{array}{c}\text { Reason } \\
\text { for } \\
\text { discontinuation }\end{array}$ & $\begin{array}{l}\text { Grade 3-4 } \\
\text { toxicity }\end{array}$ & $\begin{array}{l}\text { Modffied } \\
\text { IWG } \\
\text { response }\end{array}$ & $\begin{array}{c}\% \\
\text { decrease } \\
\text { in palpable } \\
\text { splenomegaly }\end{array}$ \\
\hline ET-1 & 54 & ET & $\begin{array}{c}\text { Anagrelide, } \\
\text { hydrea }\end{array}$ & $\begin{array}{c}\text { None } \\
\text { detected }\end{array}$ & 24 & $\begin{array}{c}\text { Trial } \\
\text { discontinuation }\end{array}$ & $\begin{array}{c}\text { Nausea } \\
\text { vomiting, } \\
\text { CPK increase }\end{array}$ & $\begin{array}{c}\text { Stable } \\
\text { disease }\end{array}$ & N/A \\
\hline PV-1 & 57 & PV & Hydrea & $J A K 2 \mathrm{~V} 617 \mathrm{~F}$ & 5.5 & $\begin{array}{l}\text { Investigator } \\
\text { decision }\end{array}$ & $\begin{array}{c}\text { Blurred } \\
\text { vision, } \\
\text { night blindness, } \\
\text { GI bleeding }\end{array}$ & $\begin{array}{c}\text { No } \\
\text { response }\end{array}$ & $33 \%$ \\
\hline MF-1 & 56 & Post-ET MF & $\begin{array}{l}\text { Hydrea, } \\
\text { anagrelide, }\end{array}$ & $\begin{array}{l}\text { JAK2V617F, } \\
\text { ASXL1P722fs }\end{array}$ & 4 & $\begin{array}{c}\text { Allogeneic } \\
\text { stem cell transplant }\end{array}$ & N/A & Stable disease & $65 \%$ \\
\hline
\end{tabular}

pomalidomide,

azacitidine

\begin{tabular}{|c|c|c|c|c|c|c|c|c|c|}
\hline MF-2 & 53 & Post-ET MF & $\begin{array}{l}\text { Anagrelide, } \\
\text { hydrea }\end{array}$ & JAK2V617F & 3.5 & $\begin{array}{c}\text { Trial } \\
\text { discontinuation }\end{array}$ & $\begin{array}{c}\text { Night } \\
\text { blindness }\end{array}$ & $\begin{array}{c}\text { Clinical } \\
\text { improvement, } \\
\text { anemia response }\end{array}$ & $42 \%$ \\
\hline MF-3 & 72 & PMF & Darbepoetin & $\begin{array}{l}\text { JAK2V617F } \\
\text { FLT3R773G } \\
\text { CEBPAP204S }\end{array}$ & 6 & $\begin{array}{c}\text { Investigator } \\
\text { decision }\end{array}$ & $\begin{array}{c}\text { GI } \\
\text { bleeding }\end{array}$ & $\begin{array}{l}\text { Stable } \\
\text { disease }\end{array}$ & $100 \%$ \\
\hline MF-4 & 54 & $\begin{array}{c}\text { PMF/ } \\
\text { MPN-AP }\end{array}$ & N/A & $\begin{array}{c}\text { DNMT3AR882P } \\
\text { SRSF2P95H }\end{array}$ & 4 & $\begin{array}{c}\text { Investigator } \\
\text { decision }\end{array}$ & $\begin{array}{l}\text { Anemia, } \\
\text { GI bleeding }\end{array}$ & $\begin{array}{l}\text { Stable } \\
\text { disease }\end{array}$ & N/A \\
\hline MF-5 & 63 & Post-PV MF & $\begin{array}{l}\text { Hydrea, } \\
\text { interferon }\end{array}$ & JAK2V617F & 0.75 & $\begin{array}{c}\text { Trial } \\
\text { discontinuation }\end{array}$ & $\begin{array}{l}\text { Anemia, } \\
\text { mbocytopen }\end{array}$ & N/A & $\mathrm{N} / \mathrm{A}$ \\
\hline
\end{tabular}

fatigue

IWG: International Working Group; MPN: myeloproliferative neoplasms; ET: essential thrombocythemia; PV: polycythemia vera; MF: myelofibrosis; PMF: primary myelofibrosis; MPN-AP: MPN accelerated phase; GI: gastrointestinal; CPK: creatine phosphokinase; N/A: not available. 
patients were enrolled on therapy after obtaining approval from Memorial Sloan Kettering Cancer Center's Institutional Review Board. MF, PV and ET were diagnosed according to World Health Organization 2008 diagnostic criteria. The primary objective of the study was to determine the efficacy of AUY922 in this patient population. Response was assessed using the Revised International Working Group for Myeloproliferative
Neoplasms Research and Treatment (IWG-MRT) and European LeukemiaNet (ELN) response criteria ${ }^{9}$ for $\mathrm{MF}$, and the European LeukemiaNet (ELN) consensus criteria ${ }^{10}$ for PV and ET. Response was assessed after six months of therapy or earlier if treatment was discontinued. Spleen response assessment was carried out by palpation below the costal margin in the midclavicular line. Patients were examined by the same physician-investigator at all

A
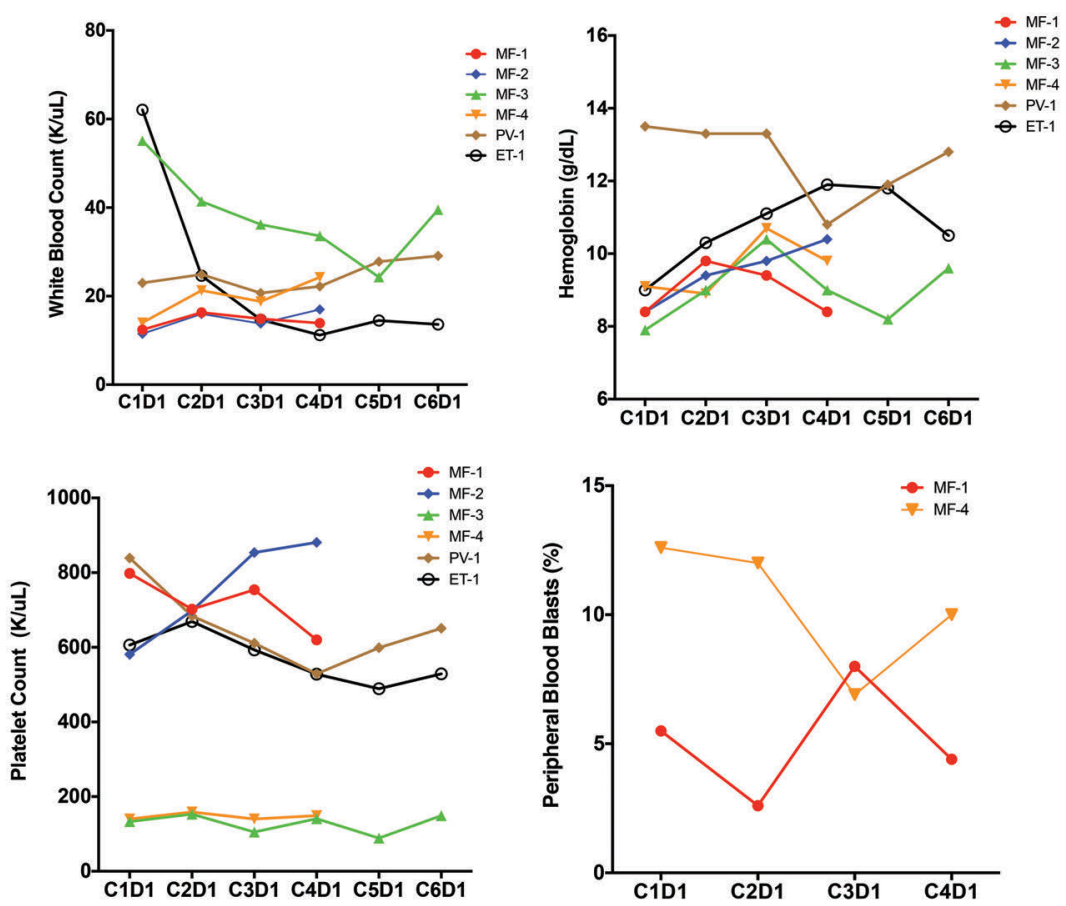

B
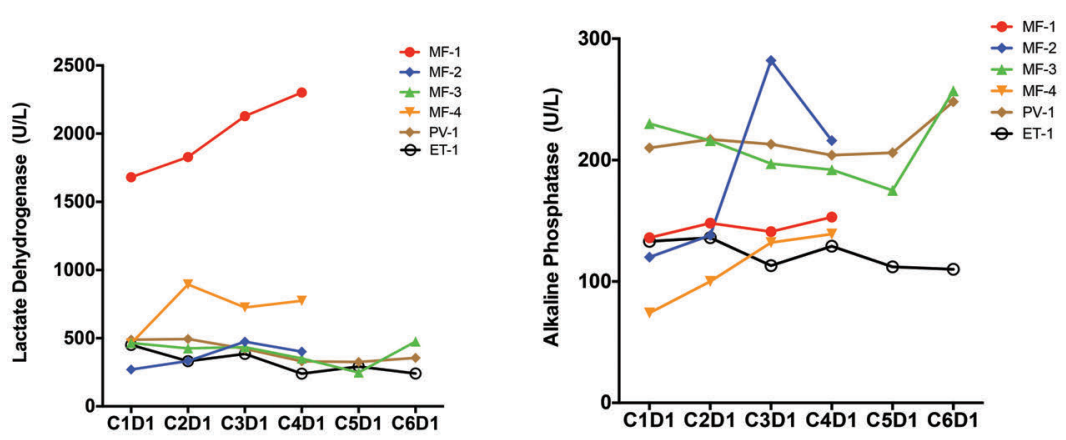

C

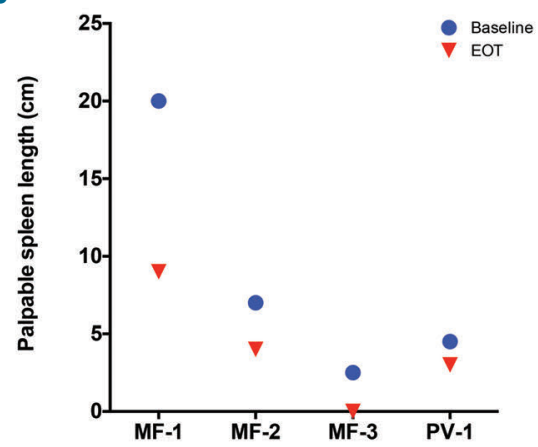

Figure 1. Impact of AUY922 treatment on clinical parameters. (A) Peripheral blood counts for treated subjects in cycles 1-6 or until time of withdrawal from study if before cycle 6 , including white blood count, hemoglobin, platelet count and peripheral blood blast count. (B) Lactate dehydrogenase and alkaline phosphatase levels in cycles 1-6. (C) Measurement of spleen length by palpation in the midclavicular line below the left costal margin at time of baseline and end of treatment (EOT) assessments. 
response time points to ensure measurement precision. Secondary objectives included confirmation of safety and tolerability along with exploration of pharmacodynamic response. AUY922 was administered via intravenous infusion at a dose of $70 \mathrm{mg} / \mathrm{m}^{2}$ once weekly. Dose reduction to $55 \mathrm{mg} / \mathrm{m}^{2}$ was permitted for patients experiencing a grade 3 or higher non-hematologic toxicity or grade 4 hematologic toxicity. Western blot analysis on peripheral blood derived granulocytes was carried out as previously described to assess JAK-STAT signaling. ${ }^{6}$ Mutational profiling was carried out using a next-generation sequencing panel as previously described. ${ }^{11}$ Peripheral blood cytokine analysis was carried out using multiplex bead assay (HCYTMAG-60K-PX38, Millipore) as previously described. $^{12}$

Twenty-five patients were considered for enrollment on study. However, due to unanticipated toxicity, only 7 patients were accrued prior to study termination. The 7 patients treated on study included: 5 with MF (including one patient with accelerated phase disease; $>10 \%$ circulat- ing blasts), and one patient each with PV and ET (Table 1). Patients were treated for a median of four months (range 0.75-24 months). Three patients with MF experienced stable disease by IWG criteria; this includes one patient with accelerated phase disease (Table 1). One MF patient met criteria for Clinical Improvement due to anemia response (2 $\mathrm{g} / \mathrm{dL}$ increase in hemoglobin from baseline to end of study without transfusion support) (Figure 1A). One MF patient did not undergo response assessment as treatment was discontinued prior to completion of cycle 1 of therapy (due to study discontinuation). Patient ET-1 experienced stable disease by ELN criteria. ${ }^{9}$ A patient with PV did not meet criteria for a response by ELN criteria.

Five of 7 patients enrolled on study had palpable splenomegaly at baseline. All patients with palpable splenomegaly experienced a reduction in palpable splenomegaly on treatment (median reduction $54 \%$, range $33-$ $100 \%$ ) (Figure 1C). These responses were not characterized by IWG criteria as imaging studies were not used to assess spleen size. All subjects, except for MF-3, were immediate-
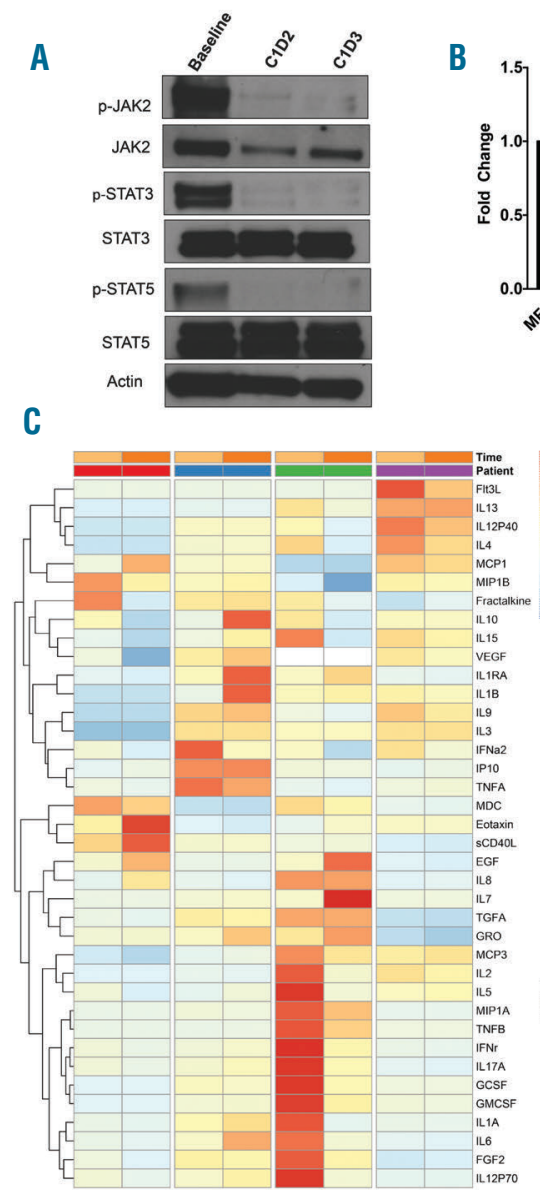

\section{D}
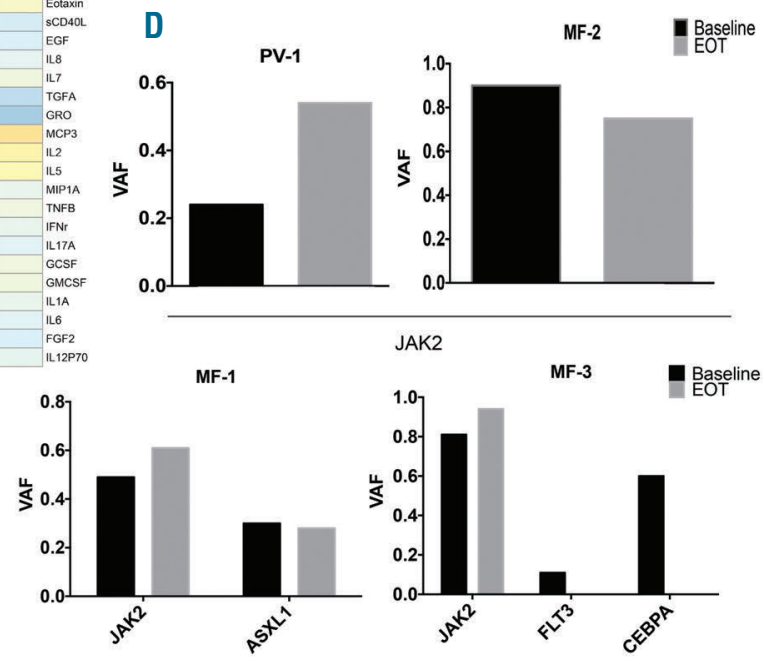

Figure 2. Impact of AUY922 treatment on pharmacodynamic parameters. (A) Representative Western blot analysis of peripheral blood granulocyte lysate at baseline (prior to drug infusions), one day post infusion (C1D2) and two days post infusion (C1D3) of patient MF-5. (B) Densitometric analysis of Western blots performed on study patients at baseline and one day post infusions (C1D2). Bar graphs display fold change compared to baseline. (C) Peripheral blood cytokine analysis performed at baseline (pre) and cycle 4 day 1 (post) infusion. Heat map depicts row normalized z-scores of cytokine abundance. (D) Variant allele fraction (VAF) based on nextgeneration sequencing performed on peripheral blood granulocytes at baseline and end of treatment (EOT). Top row represents patients with only JAK2V617F mutation detected (PV-1, MF-2). Bottom row represents patients with more than one mutation detected (MF-1, MF-3). PV: polycythemia vera; MF: myelofibrosis. 
ly transitioned to alternative therapies at the end of study. Notably, spleen again became palpable $(1-2 \mathrm{~cm})$ in MF-3 approximately two months after discontinuing therapy with AUY922.

Adverse events (grade 2 and higher) included diarrhea, night blindness, alkaline phosphatase elevation, nausea, emesis, blurred vision, and creatine phosphokinase elevation. Serious Adverse Events (SAEs) included nausea, vomiting, diarrhea, and altered mental status. Grade 3 decreased visual acuity and night blindness was observed in 2 patients, requiring dose reduction (Table 1). Three patients experienced gastrointestinal bleeding as an SAE (grade 3). Colonoscopic evaluation revealed the presence of an ileocecal ulcer in 2 of the 3 patients with gastrointestinal bleeding. Two events were deemed possibly related to exposure to the study drug. The trial was terminated for this reason.

Pre-clinical studies indicate that Hsp90 inhibition leads to decreased JAK2 protein levels and disruption of JAK-STAT signaling. In order to assess these pharmacodynamic parameters, Western blot analysis was carried out on peripheral blood granulocyte lysates collected at baseline (prior to infusion) and for two consecutive days post infusion. Analysis of JAK-STAT pathway activation by Western blot using granulocyte lysate demonstrated a marked reduction in levels of total-JAK2 and phosphorylated-JAK2 on cycle 1 day 2 and day 3 , when compared with pre-infusion levels. Concomitant reductions in phosphorylatedSTAT3 and phosphorylated-STAT5 were also observed, indicating that treatment with AUY922 was successfully able to disrupt JAK-STAT signaling (Figure 2A). Similarly, evaluation of JAK-STAT signaling in 4 patients (MF-1, MF2, MF-4 and PV-1) demonstrated reduction in levels of phosphorylated-STAT3 and reductions in total-JAK2 and phosphorylated-STAT5 in 4 out of 5 patients evaluated (Figure 2B).

Assessment of the impact of treatment with AUY922 on peripheral blood cytokine production was carried out prior to first infusion of AUY922 (cycle 1 day 1 ) and on day 1 of cycle 4. Dynamic changes were observed in several patients with elevated levels of cytokines at baseline, as well as increases in several cytokines (Figure 2C). No consistent pattern of cytokine alteration was observed, either at baseline or as a result of treatment, in this cohort of patients.

Molecular genetic profiling of patients at baseline and the end of treatment largely revealed modest changes in JAK2V617F allele burden, with increases (MF-1, PV-1) and decreases (MF-2) in allele burden noted. Variants in FLT3 and CEBPA identified in MF-3 at baseline were no longer detectable at the end of treatment (Figure 2D). Changes in allele burden did not correlate with the observed changes in spleen size. However, a decrease in the JAK2V617F allele burden was observed in a patient with an anemia response and reduction in spleen size (MF-2).

Our pilot clinical trial of AUY922 in patients with MPNs demonstrated clinical responses, including reductions in spleen size in all patients with baseline palpable splenomegaly, and an anemia response in one MF patient. Most patients treated had stable disease, including a patient with accelerated phase disease. Importantly, pharmacodynamic analysis demonstrated a decrease in JAK2 protein levels as well as inhibition of the JAK-STAT signaling pathway, as predicted by pre-clinical studies. It is not clear if JAK-STAT signaling remains disrupted for the entire period between doses, which might mitigate the extent of response and potential for consistent $J A K 2$ allele burden decreases. Further, most patients received less than six months of therapy, which may have attenuated potential efficacy. Many toxicities observed in this study are likely specific to AUY922 (based on prior clinical experience ${ }^{13,14}$ ) and not specific to Hsp90 inhibition, as data from a phase I study of the Hsp90 inhibitor PU-H71 did not demonstrate gastrointestinal bleeding or night blindness. ${ }^{15}$ Collectively, our pre-clinical and clinical data indicate that $\mathrm{Hsp} 90$ inhibition has activity in MPNs. Optimal dosing of Hsp90 inhibitors will require detailed analysis of pharmacodynamic response over time following drug exposure to ensure consistently stable disruption of JAK-STAT signaling. In addition, combination therapy with JAK inhibitors and Hsp90 inhibitors may represent a viable therapeutic strategy given the complementary mechanisms of action of these inhibitors, particularly in patients resistant to JAK inhibitor monotherapy. ${ }^{4,15}$ Further therapeutic efforts targeting Hsp90 warrant investigation in this patient population.

\section{Gabriela S. Hobbs, ${ }^{1}$ Amritha Varshini Hanasoge} Somasundara, ${ }^{2}$ Maria Kleppe, ${ }^{2}$ Rivka Litvin, ${ }^{3}$ Maria Arcila, ${ }^{4}$ Jihae Ahn, ${ }^{5}$ Anna Sophia McKenney, Kristina Knapp, Ryan Ptashkin, ${ }^{4}$ Howard Weinstein, ${ }^{8}$ Murk-Hein Heinemann, ${ }^{9}$ Jasmine Francis, ${ }^{9}$ Suzanne Chanel, ${ }^{3}$ Ellin Berman, ${ }^{3}$ Michael Mauro, ${ }^{3}$ Martin S. Tallman, ${ }^{3}$ Mark L. Heaney, Ross L. Levine, ${ }^{2,3}$ and Raajit K. Rampal ${ }^{3}$

${ }^{1}$ Division of Hematology/Oncology, Massachusetts General Hospital, Harvard Medical School; 'Human Oncology and Pathogenesis Program, Memorial Sloan Kettering Cancer Center; ${ }^{3}$ Leukemia Service, Department of Medicine, Memorial Sloan Kettering Cancer Center; ${ }^{2}$ Department of Pathology, Memorial Sloan Kettering Cancer Center; ${ }^{5}$ Driskill Graduate Program in Life Sciences, Feinberg School of Medicine, Northwestern University; ${ }^{6}$ Gerstner Sloan Kettering Graduate School of Biomedical Sciences, and Weill Cornell/Rockefeller/Sloan Kettering Tri-Institutional MD-PhD Program; ${ }^{7}$ Center for Epigenetics Research Memorial Sloan Kettering Center; ${ }^{8}$ Cardiology Service, Department of Medicine Memorial Sloan Kettering Cancer Center; Department of Surgery, Memorial Sloan Kettering Cancer Center and ${ }^{10}$ Department of Medicine, Columbia University Medical Center, USA

Funding: this work was supported by funding from Novartis Pharmaceuticals; Cancer Center Support Grant/Core Grant to Memorial Sloan Kettering Cancer Center (P30 CA008748); NCI 1K08CA188529-01 (RKR); KK12CA087723-14 (GSH); NCI R01CA173636 (RLL); K99 HL122503-01A1 (MK); NIH National Institute of General Medical Sciences Grant T32 GM007739 and NCI F30CA18349 (ASM).

Correspondence: rampalı@mskcc.org doi:10.3324/haematol.2017.177600

Information on authorship, contributions, and financial \& other disclosures was provided by the authors and is available with the online version of this article at www. haematologica.org.

\section{References}

1. Harrison C, Kiladjian JJ, Al-Ali HK, et al. JAK inhibition with ruxolitinib versus best available therapy for myelofibrosis. N Engl J Med. 2012;366(9):787-798.

2. Verstovsek S, Kantariian H, Mesa RA, et al. Safety and efficacy of INCB018424, a JAK1 and JAK2 inhibitor, in myelofibrosis. N Engl J Med. 2010;363(12):1117-1127

3. Verstovsek S, Mesa RA, Gotlib J, et al. A double-blind, placebo-controlled trial of ruxolitinib for myelofibrosis. N Engl J Med. 2012; 366(9):799-807.

4. Koppikar P, Bhagwat N, Kilpivaara O, et al. Heterodimeric JAK-STAT activation as a mechanism of persistence to JAK2 inhibitor therapy. Nature. 2012;489(7414):155-159.

5. An WG, Schulte TW, Neckers LM. The heat shock protein 90 antagonist geldanamycin alters chaperone association with p210bcr-abl and v-src proteins before their degradation by the proteasome. Cell Growth Differ. 2000;11(7):355-360.

6. Marubayashi S, Koppikar P, Taldone T, et al. HSP90 is a therapeutic target in JAK2-dependent myeloproliferative neoplasms in mice and 
humans. J Clin Invest. 2010;120(10):3578-3593.

7. Fiskus W, Verstovsek S, Manshouri T, et al. Heat shock protein 90 inhibitor is synergistic with JAK2 inhibitor and overcomes resistance to JAK2-TKI in human myeloproliferative neoplasm cells. Clin Cancer Res. 2011;17(23):7347-7358.

8. Cervantes F, Dupriez B, Pereira A, et al. New prognostic scoring system for primary myelofibrosis based on a study of the International Working Group for Myelofibrosis Research and Treatment. Blood. 2009;113(13):2895-2901.

9. Tefferi A, Cervantes F, Mesa R, et al. Revised response criteria for myelofibrosis: International Working Group-Myeloproliferative Neoplasms Research and Treatment (IWG-MRT) and European LeukemiaNet (ELN) consensus report. Blood. 2013;122(8):1395-1398.

10. Barosi G, Birgegard G, Finazzi G, et al. Response criteria for essential thrombocythemia and polycythemia vera: result of a European
LeukemiaNet consensus conference. Blood. 2009;113(20):4829-4833.

11. Cheng DT, Cheng J, Mitchell TN, et al. Detection of mutations in myeloid malignancies through paired-sample analysis of microdroplet-PCR deep sequencing data. J Mol Diagn. 2014;16(5):504-518.

12. Kleppe M, Kwak M, Koppikar P, et al. JAK-STAT pathway activation in malignant and nonmalignant cells contributes to MPN pathogenesis and therapeutic response. Cancer Discov. 2015;5(3):316-331.

13. Doi T, Onozawa Y, Fuse N, et al. Phase I dose-escalation study of the HSP90 inhibitor AUY922 in Japanese patients with advanced solid tumors. Cancer Chemother Pharmacol. 2014;74(3):629-636.

14. Sessa C, Shapiro GI, Bhalla KN, et al. First-in-human phase I doseescalation study of the HSP90 inhibitor AUY922 in patients with advanced solid tumors. Clin Cancer Res. 2013;19(13):3671-3680.

15. Gerecitano J MS, Rampal R, et al. Phase I trial of the HSP-90 inhibitor PU-H71. J Clin Oncol. 2015;33 Suppl: Abstract 2537. 